\title{
Chemoenzymatic Total Synthesis of $(+)$-Oxycodone from Phenethyl Acetate
}

\author{
Mary Ann A. Endoma-Arias \\ Mariia Makarova \\ Helen E. Dela Paz \\ Tomas Hudlicky* (D)
}

Chemistry Department and Centre for Biotechnology, Brock University, 1812 Sir Isaac Brock Way St. Catharines, Ontario, L2S 3A1, Canada

thudlicky@brocku.ca

Published as part of the 50 Years SYNTHESIS - Golden Anniversary Issue

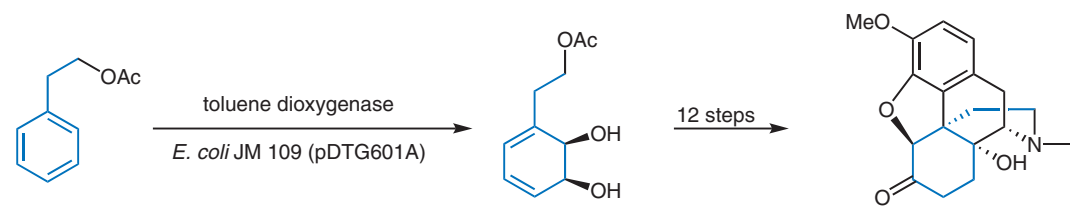

(+)-oxycodone
Received: 29.10 .2018
Accepted: 31.10 .2018
Published online: 20.11 .2018
DOI: $10.1055 / s-0037-1611335$; Art ID: ss-2018-z0729-fa
License terms: CCC

Abstract The stereoselective total synthesis of unnatural (+)-oxycodone from phenethyl acetate is described. Absolute stereochemistry was established via microbial dihydroxylation of phenethyl acetate with the recombinant strain JM109 (pDTG601A) to the corresponding ciscyclohexadienediol whose configuration provides for the absolute stereochemistry of the ring $\mathrm{C}$ of $(+)$-oxycodone. Intramolecular Heck cyclization was employed to establish the quaternary carbon at C-13, along with the dibenzodihydrofuran functionality. The C-14 hydroxyl was installed via $\mathrm{Sml}_{2}$-mediated radical cyclization. The synthesis of (+)-oxycodone was completed in a total of 13 steps and an overall yield of $1.5 \%$. Experimental and spectral data are provided for all new compounds.

Key words enzymatic dihydroxylation, total synthesis, oxycodone, Parker's hydroamination, pinacol-type coupling

The semi-synthetic opioid (-)-oxycodone (1) (Figure 1), although found also in nature, ${ }^{1}$ is a potent analgesic that is clinically prescribed for pain management. ${ }^{2}$ It is taken by mouth and is available mixed with acetaminophen in immediate release tablet form, which contains oxycodone $\mathrm{HCl}$ $(5 \mathrm{mg})$ and acetaminophen $(325 \mathrm{mg})\left(\right.$ Percocet $\left.^{\circledR}\right),{ }^{3}$ as a single ingredient medication oxycodone $\mathrm{HCl}(60 \mathrm{mg}$ and 80 $\mathrm{mg}$ ) or as film coated, extended release tablet OxyContin ${ }^{\circledR} .4$ The commercial route ${ }^{5}$ for the preparation of oxycodone is a two-step process from thebaine through the oxidation of the diene moiety with a peroxy acid to form an enone followed by hydrogenation. ${ }^{6}$ Thebaine is a minor constituent of opium and thus this fact limits the production of oxycodone. However, thebaine, as well as oripavine, are now also available from genetically modified poppies that produce much higher percentages of these alkaloids. ${ }^{7}$ These compounds are now supplied by Tasmanian Alkaloids, Inc. ${ }^{8}$
During the past 70 years, since the milestone synthesis of morphine by Gates, ${ }^{9}$ there have been more than 30 total syntheses of morphine and related alkaloids and the academic effort continues unabated. ${ }^{10}$ Even the most efficient synthesis reported by Rice ${ }^{11}$ may not be suitable for scaleup in the industrial preparation of morphinans. Although the development of a truly practical total synthesis of any morphinan or an opiate-derived agent on a commercial scale seems like a distant dream we have attempted to design a method for the synthesis of oxycodone from readily available starting materials. A de novo preparation of oxycodone or any other medicinal opiate-derived agents for medicinal use may serve as an insurance against any future unforeseen events that may limit the supply of natural sources because of climate or political instabilities in the opium-producing regions. To date, there is only one published total synthesis of oxycodone. ${ }^{12}$ Fukuyama and coworkers accomplished the total synthesis of (-)-oxycodone (1) from 2-bromoisovanillin in 24 steps in an overall yield of $0.016 \%$. The key steps in their synthesis featured a direct intramolecular arylation of an aryl bromide, an oxidative dearomatization reaction, an intramolecular Michael addition, and a Hofmann rearrangement. Absolute stereochemistry was incorporated into the starting material by the use of Evans' oxazolidinone as a chiral auxiliary.

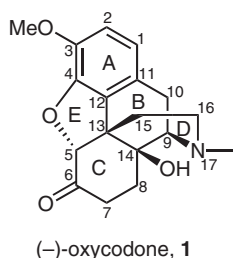

Figure 1 Structure of (-)-oxycodone (with numbering system shown) 


\section{Biographical sketches}
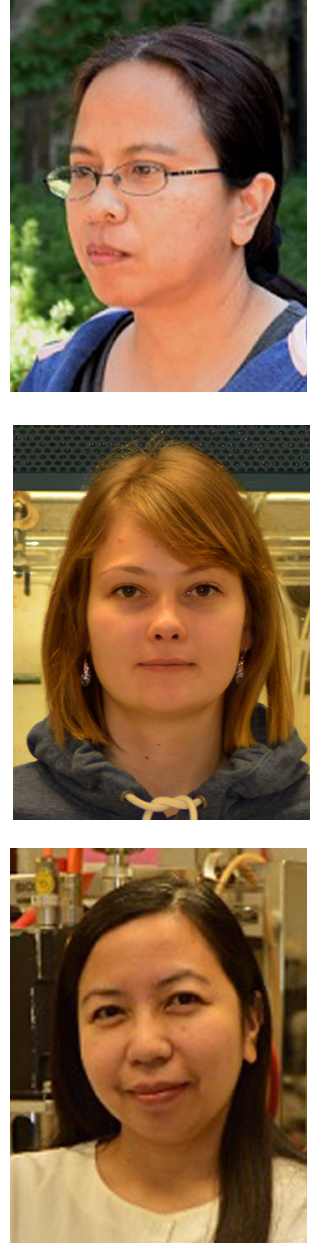

Helen E. Dela Paz was born in 1975 in Rizal, Philippines. She obtained her B.Sc. in Food Technology at the University of the Philippines Diliman, in Quezon City, Philippines. She was given the opportunity to apply her knowledge of microbiology to fermentation technology. Thus, in 2001 , she was offered a research position in the laboratories of Dr. Tomas Hudlicky at the University of

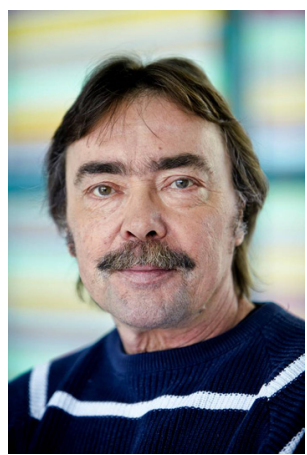

Tomas Hudlicky was born in 1949 in Prague, Czechoslovakia, where he received his elementary and middle school education. After several years of working as a process chemist apprentice and in other odd jobs in pharmaceutical chemistry, it became apparent that higher education opportunities were closed to him. In 1968, he emigrated to the U.S. with his parents and sister. Hudlicky's educational experience continued at Blacksburg High School, from which he dropped out in the spring of 1969. Accepted as a probational student at Virginia Tech the following autumn, he received his B.S. in chemistry in 1973, and went on to pursue graduate studies at Rice University under the direction of Professor Ernest Wenkert in the field of indole alkaloid total syn-
$\mathrm{PhD}$ in 1997. Upon completion of her PhD, she returned to the University of the Philippines where she rose to the rank of Associate Professor. Her main interest during her research career in the Philippines focused on the synthesis and conjugation of compounds with medicinal properties to liposomes and hydrophilic polymers for targeted delivery. During this time she continued to work closely with the

ies at Brock University, St. Catharines, Canada under the supervision of Professor Tomas Hudlicky. During her second year of M.Sc. she transferred to the Ph.D. program.
Hudlicky Research Group. Since 2010 she has been working with the Research group of Professor Hudlicky at Brock University in St. Catharines, ON, Canada as a Research Associate. In 2015 she emigrated to Canada with her husband and children. Her main research interest is the total synthesis of morphine and related alkaloids. To date, she has published 30 papers and 5 patents.

Her Ph.D. research has been focused on the chemoenzymatic approach towards synthesis of morphine alkaloids. Her defense is scheduled on November 30, 2018.
Florida, Gainesville, FL, USA. While at UF, she was introduced to the utility of a fermentor in the large scale microbial oxidation of aromatic compounds towards the production of value-added metabolites. After almost two years of work, she returned to the Philippines and took a job as a Science teacher. In 2014, she returned to the Hudlicky Research Group at Brock University to work as

thesis, earning his Ph.D. in 1977. He then spent a year at the University of Geneva working under the late Professor Wolfgang Oppolzer on the synthesis of isocomene. In 1978, he joined the faculty at the Illinois Institute of Technology as an Assistant Professor, and began the first phase of his research career in the field of general methods of synthesis for triquinane terpenes and other natural products containing five-membered rings by $[4+1]$ cyclopentene, pyrroline, and dihydrofuran annulation methodologies. He returned to his alma mater, Virginia Tech, in 1982, and rose to the rank of Professor in 1988. One year later, at the 20year class reunion of the Blacksburg High School class of 1969, he received his High School Diploma. The next phase of his research involved a Researcher and join her sister who has been working there. Her research interest focuses on the production of known diene diols from aromatic compounds, and the identification of new ones. She has published a few papers related to biocatalysis and its application to organic synthesis.

the investigation of cis-cyclohexadienediols in enantioselective synthesis. In 1995, he moved to University of Florida in Gainesville. In 2003, Dr. Hudlicky accepted an offer from Brock University where he currently holds a position as Canada Research Chair. His current research interests include the development of enantioselective synthetic methods, bacterial dioxygenase-mediated degradation of aromatics, design and synthesis of fluorinated inhalation anesthetic agents, synthesis of morphine and Amaryllidaceae alkaloids, and design of unnatural oligosaccharide conjugates with new molecular properties. His hobbies are skiing, hockey, martial arts, and music 
Scheme 1 outlines our retrosynthesis of (+)-oxycodone. Disconnection of ring $\mathrm{D}$ leads to styrene $\mathbf{2}$. In the forward sense, Parker's hydroamination can be utilized to construct the C-9 stereogenic center. The tosylamide group necessary for the cyclization reaction is derived from acetate $\mathbf{3}$, which is envisioned to be prepared from the keto acetal $\mathbf{4}$ following a deprotection of acetal and a $\mathrm{SmI}_{2}$-mediated pinacoltype coupling reaction. The key intermediate $\mathbf{4}$ can be obtained from alkene $\mathbf{5}$ via dihydroxylation followed by selective mesylation of the less hindered hydroxyl group and the elimination of the mesylate to reveal the ketone functionality in $\mathbf{4}$. Alkene $\mathbf{5}$ can be obtained in two steps from alcohol 7 via a sequence of steps that involves a Mitsunobu coupling with an iodophenol acetal to furnish aryl ether 6 followed by an intramolecular Heck reaction. The absolute stereochemistry in $\mathbf{7}$ is incorporated via microbial dihydroxylation with toluene dioxygenase, overexpressed in E.coli JM109 (pDTG601A), in the whole-cell fermentation of phenethyl acetate $(\mathbf{8}) .{ }^{13}$ The enzymatically derived arene cis-dihydrodiols such as $\mathbf{7}$ have found widespread use in enantioselective synthesis of natural products. ${ }^{14}$

The synthesis began with the microbial dihydroxylation of phenethyl acetate (8) (Scheme 2) in a whole cell fermentation with E. coli JM109 (pDTG601A) to afford the intermediate cyclohexadiene diol 7 (obtained in $5 \mathrm{gL}^{-1}$ yield), ${ }^{15}$ which was subjected to a selective reduction of the less hindered alkene to afford the known diol $\mathbf{9}^{16}$ (85\% yield). The distal, less hindered, hydroxyl in diol $\mathbf{9}$ was protected with tert-butyldimethylsilyl chloride and the proximal allylic alcohol was then coupled with iodophenol $\mathbf{1 0},{ }^{17}$ derived from isovanillin, via a Mitsunobu reaction to furnish ether $\mathbf{6}(45 \%$ yield over two steps). A subsequent intramolecular Heck reaction of $\mathbf{6}$ produced olefin $\mathbf{5}$ (87\% yield) whose dihydroxylation led to diol 11 ( $81 \%$ yield). This compound possesses the features of the ACE rings of oxycodone. The diol functionality was converted to ketone $\mathbf{4}$ via mesylation of the less hindered hydroxyl group followed by DBU-catalyzed elimination of the resulting mesylate (63\% yield over two steps). With the attainment of $\mathbf{4}$, deprotection of the acetal followed by a pinacol-type coupling of the intermediate keto aldehyde using $\mathrm{SmI}_{2}$ was conducted to afford diol 3, tentatively assigned as the cis-isomer (65\% yield over two steps). ${ }^{18}$ Protection of diol 3 with a carbonate group to afford 12 ( $80 \%$ yield) allowed for the introduction of the tosylamide functionality via methanolysis of the acetate followed by Mitsunobu coupling of the resulting alcohol with $N$-methyl $p$-toluenesulfonyl amide. The carbonate moiety in the crude tosylamide was hydrolyzed to afford diol 13 (67\% yield over three steps). It would have been desirable to isolate the intermediate tosylamide carbonate but the difficulty in the separation of residual $\mathrm{N}$-methyl $p$-toluenesulfonyl amide from the desired product rendered this process impractical. The less hindered hydroxyl of 13 was converted to the corresponding mesylate and subjected to DBU-catalyzed elimination affording alkene 2 (70\% yield over two steps), which was the precursor for the key Parker's hydroamination step to complete the ring system of oxycodone. Thus, treatment of $\mathbf{2}$ with $\mathrm{Li}$ in liquid ammonia as reported by Parker ${ }^{19}$ in the total synthesis of hydrocodone afforded the oxycodol ether 14 (76\% yield). ${ }^{20}$ Deprotection of the TBS group in $\mathbf{1 4}$ followed by oxidation of the alcohol to ketone afforded ent-oxycodone [ent-(1)] (59\% yield over two steps).

In conclusion, a short chemoenzymatic synthesis of entoxycodone has been accomplished in 13 steps from phenethyl acetate. Further improvements in this short synthesis will address installation of the $\mathrm{N}$-methyltosylamide side chain earlier in the synthesis thus eliminating the need to use a carbonate protecting group during the synthetic sequence. In particular, the acetate functionality in $\mathbf{5}$ will be converted to the tosylamide. Furthermore, an $\mathrm{SmI}_{2}$-mediated nitrone-keto coupling is being investigated to afford an amino alcohol instead of diol intermediate for subsequent cyclization to the pendant acetate side chain. These

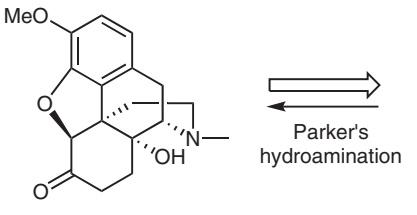

(+)-oxycodone [ent-(1)]

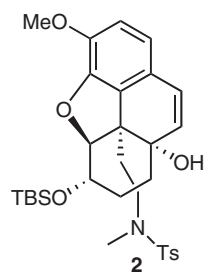

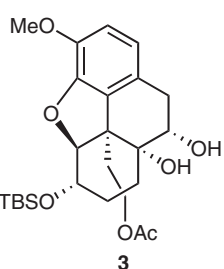

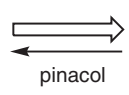

tosylamidationelimination

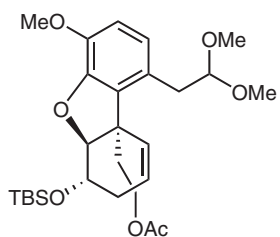

5

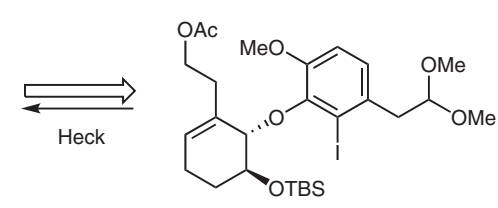

6

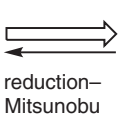

Mitsunobu

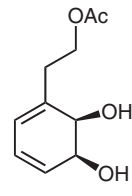

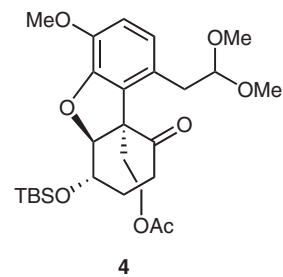

4

Scheme 1 Retrosynthetic analysis of the route to ent-oxycodone 
<smiles>CC(=O)OCCc1ccccc1</smiles><smiles>CC(=O)OCCC1=CC=CC(O)C1O</smiles>

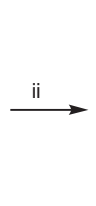<smiles>CC(=O)OCCC1=CCCC(O)C1O</smiles>

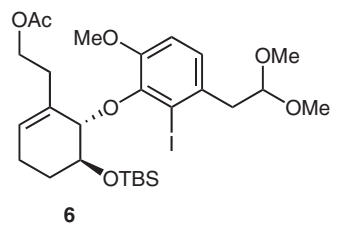

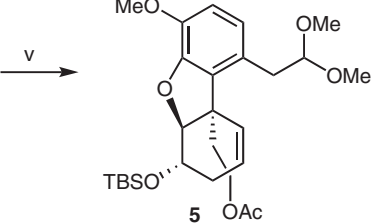

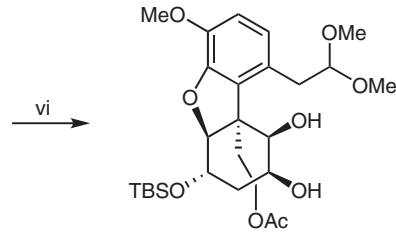

11<smiles>CCCCC</smiles><smiles>COc1ccc(CC(OC)OC)c2c1O[C@@H]1C(CC(OC)C[C@H]1C(C)C)C2=O</smiles><smiles>C=CC</smiles>

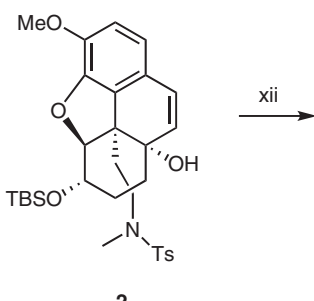

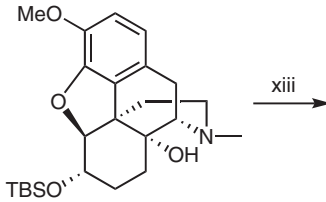

14

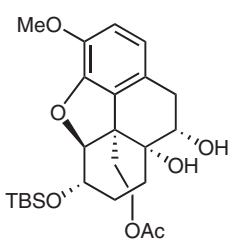

3

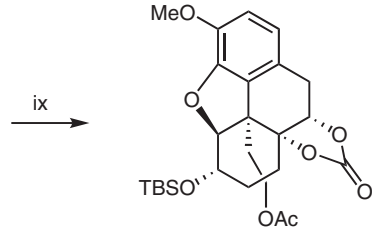

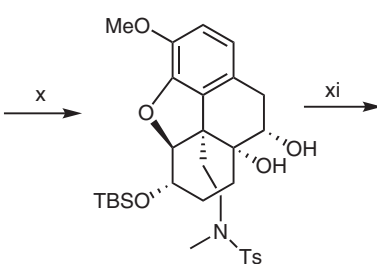

13
2<smiles>CNC1Cc2ccc(OC)c3c2C2(CCCNC2)[C@H]1O3</smiles>

(+)-oxycodone [ent-(1)]<smiles>COc1ccc(CC(C)OC)c(C)c1O</smiles>

Scheme 2 Synthesis of ent-oxycodone. Reagents and conditions: i) E. coli JM 109 (pDTG601A), $5 \mathrm{gL}^{-1}$; ii) potassium azodicarboxylate, MeOH, AcOH, 85\%; iii) TBSCl, imidazole, $\mathrm{CH}_{2} \mathrm{Cl}_{2}, 54 \%$; iv) 10 , $\mathrm{TMAD}, \mathrm{n}-\mathrm{Bu}_{3} \mathrm{P}, \mathrm{THF}, 0{ }^{\circ} \mathrm{C}$ to r.t., $83 \%$; v) $\mathrm{Ag}_{2} \mathrm{CO}_{3}$, dppp, $\mathrm{Pd}(\mathrm{OAc})_{2}$, DMF, reflux, $87 \%$; vi) cat. OsO $\mathrm{O}_{4}$, NMO, acetone/ $\mathrm{H}_{2} \mathrm{O}, 81 \%$; vii) a. $\mathrm{MsCl}, \mathrm{NEt}_{3}, \mathrm{CH}_{2} \mathrm{Cl}_{2}$, b. DBU, toluene, reflux, $63 \%$ over 2 steps; viii) a. aq TFA, toluene, $50{ }^{\circ} \mathrm{C}$, b. $\mathrm{Sml}{ }_{2}, \mathrm{THF},-78{ }^{\circ} \mathrm{C}, 65 \%$ over 2 steps; ix) carbonyldiimidazole, toluene, $80{ }^{\circ} \mathrm{C}, 80 \%$; $x$ ) a. $\mathrm{K}_{2} \mathrm{CO}_{3}$, MeOH, b. TsNHMe, TMAD, $n-\mathrm{Bu}_{3} \mathrm{P}$, THF, c. aq $\mathrm{NaOH}, \mathrm{MeOH}, 67 \%$ over 3 steps; $\mathrm{xi}$ ) a. $\mathrm{MsCl}$, $\mathrm{NEt}{ }_{3}$, $\mathrm{CH}_{2} \mathrm{Cl}_{2}$, b. DBU, toluene, reflux, $70 \%$ over 2 steps; xii) $\mathrm{Li}, t-\mathrm{BuOH}$, THF, liq $\mathrm{NH}_{3},-78{ }^{\circ} \mathrm{C}, 76 \%$; xiii) a. TBAF, THF, b. Dess-Martin periodinane, $\mathrm{CH}_{2} \mathrm{Cl}_{2}, 59 \%$ yield over 2 steps.

improvements, as well as the 2nd generation synthesis of the natural enantiomer, are currently being investigated and will be reported in due course.

Inoculum was obtained from viable cells stored at $-78{ }^{\circ} \mathrm{C}$ in cryovials. They were grown in suitable media as previously described. ${ }^{15 b}$ Substrate was fed in $5 \mathrm{~mL}$ increments over the course of $3 \mathrm{~h}$ with metabolites being harvested in the usual manner. All non-aqueous reactions were conducted in an argon atmosphere using standard Schlenk techniques for the exclusion of moisture and air. $\mathrm{CH}_{2} \mathrm{Cl}_{2}$ was distilled from $\mathrm{CaH}_{2}$; THF and toluene were dried over Na/benzophenone. Analytical TLC was performed on Silicycle $60 \AA 250 \mathrm{~mm}$ TLC plates with F-254 indicator. Flash column chromatography was performed using silica gel 60 (230-400 mesh). Melting points were recorded on a Hoover Unimelt apparatus and are uncorrected. IR spectra were obtained on a PerkinElmer One FT-IR spectrophotometer. Optical rotation was measured on a PerkinElmer 341 polarimeter at a wavelength of 589 $\mathrm{nm} .{ }^{1} \mathrm{H}$ and ${ }^{13} \mathrm{C}$ spectra were recorded on a $300 \mathrm{MHz}$ and $400 \mathrm{MHz}$ Bruker spectrometer. All chemical shifts are referenced to TMS or residual nondeuterated solvent. Data of proton spectra are reported as follows: chemical shift in ppm [multiplicity (standard abbreviations), coupling constants $(\mathrm{Hz})$, integration]. ${ }^{13} \mathrm{C}$ NMR spectra were recorded with complete proton decoupling and the chemical shifts are reported in $\mathrm{ppm}(\delta)$ relative to solvent resonance as internal standard. Mass spectra and high-resolution mass spectra were performed by the Analytical Division at Brock University.

\section{2-[(5S,6R)-5,6-Dihydroxycyclohexa-1,3-dien-1-yl]ethyl Acetate $(7)^{16}$}

This compound was prepared according to a literature procedure. ${ }^{16}$ $[\alpha]_{\mathrm{D}}^{20}+32.07\left(c=0.4, \mathrm{CHCl}_{3}\right)\left\{\right.$ Lit. $\left.^{16}[\alpha]_{\mathrm{D}}^{28}+40.7\left(c=2.0, \mathrm{CHCl}_{3}\right)\right\} ; R_{f}=$ 0.18 (hexanes/EtOAc 1:2).

IR $\left(\mathrm{CHCl}_{3}\right)$ : 3392, 2955, 2925, 1735, 1383, 1366, 1238, 1037, $803 \mathrm{~cm}^{-1}$. ${ }^{1} \mathrm{H}$ NMR $\left(300 \mathrm{MHz}, \mathrm{CDCl}_{3}\right): \delta=5.90(\mathrm{ddd}, J=9.5,5.2,0.9 \mathrm{~Hz}, 1 \mathrm{H}), 5.80$ (dd, $J=9.3,3.4 \mathrm{~Hz}, 1 \mathrm{H}), 5.75-5.70(\mathrm{~m}, 1 \mathrm{H}), 4.30-4.14(\mathrm{~m}, 3 \mathrm{H}), 4.09$ (d, $J=6.0 \mathrm{~Hz}, 1 \mathrm{H}), 2.97(\mathrm{~s}, 2 \mathrm{H}), 2.57-2.48(\mathrm{~m}, 2 \mathrm{H}), 2.01(\mathrm{~s}, 3 \mathrm{H})$.

${ }^{13} \mathrm{C}$ NMR $\left(75 \mathrm{MHz}, \mathrm{CDCl}_{3}\right): \delta=171.5,137.7,128.2,124.7,121.6,70.1$, $68.5,63.1,33.3,21.1$.

MS (EI): $m / z=198,120,107,91,75$.

HRMS (EI): $m / z$ calcd for $\mathrm{C}_{10} \mathrm{H}_{14} \mathrm{O}_{4}$ : 198.0892; found: 198.0889 .

\section{2-[(5S,6R)-5,6-Dihydroxycyclohex-1-enyl]ethyl Acetate $(9)^{17}$}

To a stirred mixture of diol $7(150 \mathrm{mg}, 0.76 \mathrm{mmol})$ and potassium azodicarboxylate (PAD) $(200 \mathrm{mg}, 1.03 \mathrm{mmol})$ in $\mathrm{MeOH}(4 \mathrm{~mL})$ at $0{ }^{\circ} \mathrm{C}$ was added dropwise (over $15 \mathrm{~min}) \mathrm{AcOH}(0.5 \mathrm{~mL}, 8.75 \mathrm{mmol})$ in $\mathrm{MeOH}(1 \mathrm{~mL})$. The reaction was complete after $30 \mathrm{~min}$, as monitored by TLC (2:1 EtOAc/hexanes). The mixture was concentrated via rotary evaporation to afford a crude product that was resuspended in $\mathrm{CH}_{2} \mathrm{Cl}_{2}$ $\left(10 \mathrm{~mL}\right.$ ), and the $\mathrm{CH}_{2} \mathrm{Cl}_{2}$ layer was washed with sat. aq $\mathrm{NaHCO}_{3}(5 \mathrm{~mL})$. The organic extract was dried $\left(\mathrm{MgSO}_{4}\right)$, filtered, and evaporated to remove solvent to afford a crude oily residue that was chromatographed 
on silica gel using 2:1 EtOAc/hexanes as eluent to afford 9 as an oil; yield: $128 \mathrm{mg}(85 \%) ;[\alpha]_{\mathrm{D}}{ }^{20}-65.9\left(c=1.0, \mathrm{CHCl}_{3}\right)\left\{\right.$ Lit. $^{17}[\alpha]_{\mathrm{D}}{ }^{20}-53.0(c$ $\left.\left.=0.2, \mathrm{CHCl}_{3}\right)\right\} ; R_{f}=0.25$ (hexanes/EtOAc 1:2).

${ }^{1} \mathrm{H}$ NMR $\left(300 \mathrm{MHz}, \mathrm{CDCl}_{3}\right): \delta=5.63(\mathrm{t}, J=3.7 \mathrm{~Hz}, 1 \mathrm{H}), 4.37-4.26(\mathrm{~m}, 1$ $\mathrm{H}), 4.22-4.12(\mathrm{~m}, 1 \mathrm{H}), 4.03(\mathrm{~d}, J=3.8 \mathrm{~Hz}, 1 \mathrm{H}), 3.79-3.71(\mathrm{~m}, 1 \mathrm{H})$, 2.57-2.33 (m, 3 H), 2.23-2.05 (m, $3 \mathrm{H}), 2.04(\mathrm{~s}, 3 \mathrm{H}), 1.75-1.62(\mathrm{~m}, 2$ $\mathrm{H})$.

${ }^{13} \mathrm{C}$ NMR $\left(75 \mathrm{MHz}, \mathrm{CDCl}_{3}\right): \delta=171.4,133.6,127.6,69.5,68.6,63.3$, $33.9,24.9,24.1,20.9$.

\section{2-\{(5S,6S)-5-[(tert-Butyldimethylsilyl)oxy]-6-[3-(2,2-dime-} thoxyethyl)-2-iodo-6-methoxyphenoxy]cyclohex-1-en-1-yl\}ethyl Acetate (6)

To a stirred solution of alcohol $\mathbf{9}(3.1 \mathrm{~g}, 10.0 \mathrm{mmol})$ and 3-(2,2-dimethoxyethyl)-2-iodo-6-methoxyphenol ${ }^{15}(\mathbf{1 0} ; 3.7 \mathrm{~g}, 10.9 \mathrm{mmol})$ at $-10{ }^{\circ} \mathrm{C}$ in THF $(40 \mathrm{~mL})$ was added $n \mathrm{Bu}_{3} \mathrm{P}(3.5 \mathrm{~mL}, 14.0 \mathrm{mmol})$, followed by $\operatorname{TMAD}(2.3 \mathrm{~g}, 13.0 \mathrm{mmol})$. The reaction mixture was allowed to warm up to r.t. and stirred for $16 \mathrm{~h}$ at r.t. The solvent was removed by rotary evaporation and the residue was chromatographed on silica gel using hexanes/EtOAc as eluent (4:1 to 2:1) to afford the product 6 as an oil; yield: $5.2 \mathrm{~g}(83 \%) ;[\alpha]_{\mathrm{D}}{ }^{20}+48.4\left(c=1.2, \mathrm{CH}_{2} \mathrm{Cl}_{2}\right) ; R_{f}=0.58(2: 1$ hexanes/EtOAc).

IR (film): 2930, 2855, 1738, 1642, 1472, 1249, 1078, $776 \mathrm{~cm}^{-1}$.

${ }^{1} \mathrm{H} \mathrm{NMR}\left(300 \mathrm{MHz}, \mathrm{CDCl}_{3}\right): \delta=7.01(\mathrm{~d}, J=8.4 \mathrm{~Hz}, 1 \mathrm{H}), 6.86(\mathrm{~d}, J=8.4$ $\mathrm{Hz}, 1 \mathrm{H}), 5.87(\mathrm{~d}, J=4.5 \mathrm{~Hz}, 1 \mathrm{H}), 4.58(\mathrm{~s}, 1 \mathrm{H}), 4.56(\mathrm{t}, J=5.7 \mathrm{~Hz}, 1 \mathrm{H})$, $4.21(\mathrm{~m}, 2 \mathrm{H}), 4.05(\mathrm{~m}, 1 \mathrm{H}), 3.87(\mathrm{~s}, 3 \mathrm{H}), 3.36(\mathrm{~s}, 6 \mathrm{H}), 3.09(\mathrm{~d}, J=5.7$ $\mathrm{Hz}, 2 \mathrm{H}), 2.47-2.61$ (m, $2 \mathrm{H}), 2.28-2.37$ (m, $2 \mathrm{H}), 2.04$ (s, $3 \mathrm{H}), 2.00-$ $2.06(\mathrm{~m}, 1 \mathrm{H}), 1.61-1.70(\mathrm{~m}, 1 \mathrm{H}), 0.77(\mathrm{~s}, 9 \mathrm{H}),-0.13(\mathrm{~s}, 3 \mathrm{H}),-0.18(\mathrm{~s}$, $3 \mathrm{H})$.

${ }^{13} \mathrm{C}$ NMR $\left(75 \mathrm{MHz}, \mathrm{CDCl}_{3}\right): \delta=171.1,150.2,146.8,132.9,130.4,130.0$, 125.8, 112.1, 104.5, 79.3, 67.5, 63.7, 55.5, 54.2, 54.1, 44.4, 33.7, 25.6, $21.0,20.7,17.9,-5.1,-5.2$.

MS (EI+): $m / z(\%)=657$ (100), 565 (10), 297 (10), 237 (25).

HRMS (EI+): $m / z$ calcd for $\mathrm{C}_{27} \mathrm{H}_{41} \mathrm{IO}_{7} \mathrm{Si}[\mathrm{M}-2 \mathrm{H}]$ : 632.1666; found: 632.1657 .

\section{2-[(5aS,6S,9aR)-6-(tert-Butyldimethylsilyloxy)-1-(2,2-dime- thoxyethyl)-4-methoxy-5a,6,7,9a-tetrahydrodibenzo[b,d]furan- 9a-yl]ethyl Acetate (5) ${ }^{16 a}$}

To a stirred solution of ether 6 (3.02 g, $4.76 \mathrm{mmol})$ in DMF (55 mL) was added $\mathrm{Pd}(\mathrm{OAc})_{2}(168 \mathrm{mg}, 0.71 \mathrm{mmol}), \mathrm{Ag}_{2} \mathrm{CO}_{3}(3.9 \mathrm{~g}, 14.3 \mathrm{mmol})$, and dppp (590 mg, $1.43 \mathrm{mmol}$ ). The resulting mixture was heated to reflux for $3 \mathrm{~h}$. The cooled reaction mixture was diluted with $\mathrm{Et}_{2} \mathrm{O} / \mathrm{H}_{2} \mathrm{O}$ $(100 \mathrm{~mL} / 50 \mathrm{~mL})$. The layers were separated and the aqueous phase was further extracted with $\mathrm{Et}_{2} \mathrm{O}(2 \times 50 \mathrm{~mL})$. The combined organic extracts were washed with brine, dried $\left(\mathrm{MgSO}_{4}\right)$, filtered, and concentrated to afford a residue that was chromatographed on silica gel using hexanes/EtOAc (8:1 to 4:1) as eluent to afford the product 5 as an oil; yield: $2.1 \mathrm{~g}(87 \%)$.

\section{2-\{(5aS,6S,8S,9R,9aS)-6-[(tert-Butyldimethylsilyl)oxy]-1-(2,2-di- methoxyethyl)-8,9-dihydroxy-4-methoxy-5a,6,7,8,9,9a-hexahy- drodibenzo[b,d]furan-9a-yl\}ethyl Acetate (11)}

To a stirred solution of alkene $\mathbf{5}$ ( $1.5 \mathrm{~g}, 2.95 \mathrm{mmol}$ ) in acetone $/ \mathrm{H}_{2} \mathrm{O}$ (30 $\mathrm{mL} / 9 \mathrm{~mL}$ ) was added $\mathrm{N}$-methylmorpholine oxide (NMO; $345 \mathrm{mg}, 2.95$ mmol) followed by a catalytic amount of $\mathrm{K}_{2} \mathrm{OsO}_{4} \cdot 2 \mathrm{H}_{2} \mathrm{O}$. The resulting solution was stirred at r.t. for $2 \mathrm{~d}$ whereupon the mixture was diluted with EtOAc/ $\mathrm{H}_{2} \mathrm{O}(30 \mathrm{~mL} / 15 \mathrm{~mL})$. The layers were separated and the aqueous phase was further extracted with EtOAc $(2 \times 20 \mathrm{~mL})$. The combined organic extracts were dried $\left(\mathrm{MgSO}_{4}\right)$, filtered, and concentrated to afford a residue that was chromatographed on silica gel using hexanes/EtOAc (1:1 to $1: 2)$ as eluent to afford the product $\mathbf{1 1}$ as an oil; yield: $1.3 \mathrm{~g}(81 \%) ;[\alpha]_{\mathrm{D}}{ }^{20}+16.6\left(c=16.7, \mathrm{CH}_{2} \mathrm{Cl}_{2}\right) ; R_{f}=0.36(1: 1$ hexanes/EtOAc).

IR (film): 3429, 2951, 2856, 1738, 1629, 1433, $1042 \mathrm{~cm}^{-1}$.

${ }^{1} \mathrm{H}$ NMR $\left(300 \mathrm{MHz}, \mathrm{CDCl}_{3}\right): \delta=6.72(\mathrm{~d}, J=8.4 \mathrm{~Hz}, 1 \mathrm{H}), 6.64(\mathrm{~d}, J=8.4$ $\mathrm{Hz}, 1 \mathrm{H}), 4.57(\mathrm{dd}, J=3.3,7.8 \mathrm{~Hz}, 1 \mathrm{H}), 4.50(\mathrm{dd}, J=3.6,3.6 \mathrm{~Hz}, 1 \mathrm{H})$, 3.90-3.98 (m, $2 \mathrm{H}), 3.80$ (s, $3 \mathrm{H}), 3.37(\mathrm{~s}, 3 \mathrm{H}), 3.33(\mathrm{~d}, J=3.3 \mathrm{~Hz}, 1 \mathrm{H})$, $3.25(\mathrm{~s}, 3 \mathrm{H}), 2.84-2.90(\mathrm{~m}, 2 \mathrm{H}), 2.22-2.30(\mathrm{~m}, 2 \mathrm{H}), 2.03$ (td, $J=3.3$, $13.2 \mathrm{~Hz}, 1 \mathrm{H}), 1.78(\mathrm{dt}, J=4.2,13.2 \mathrm{~Hz}, 1 \mathrm{H}), 0.85(\mathrm{~s}, 9 \mathrm{H}), 0.08(\mathrm{~s}, 3 \mathrm{H})$, $0.06(\mathrm{~s}, 3 \mathrm{H})$.

${ }^{13} \mathrm{C}$ NMR $\left(75 \mathrm{MHz}, \mathrm{CDCl}_{3}\right): \delta=170.7,148.3,143.3,128.8,125.1,121.8$, 112.0, 106.2, 86.2, 76.7, 75.1, 68.8, 64.0, 61.4, 55.8, 55.7, 53.4, 52.6, $34.5,33.0,32.1,25.7,20.8,17.9,-5.09,-5.09$.

MS (EI+): $m / z(\%)=483$ (30), 451 (25), 359 (50), 289 (100), 259 (30), 167 (50), 149 (60), 121 (40).

HRMS (EI+): $m / z$ calcd for $\mathrm{C}_{27} \mathrm{H}_{44} \mathrm{O}_{4} \mathrm{Si}$ : 540.2755; found: 540.2734 .

\section{2-\{(5aS,6S,9aR)-6-[(tert-Butyldimethylsilyl)oxy]-1-(2,2-dime- thoxyethyl)-4-methoxy-9-oxo-5a,6,7,8,9,9a-hexahydrodiben- zo[b,d]furan-9a-yl\}ethyl Acetate (4)}

To a stirred solution of diol $\mathbf{1 1}(1.41 \mathrm{~g}, 2.61 \mathrm{mmol})$ in $\mathrm{CH}_{2} \mathrm{Cl}_{2}(37 \mathrm{~mL})$ at $0{ }^{\circ} \mathrm{C}$ was added $\mathrm{NEt}_{3}(724 \mu \mathrm{L}, 5.22 \mathrm{mmol})$ followed by $\mathrm{MsCl}(303 \mu \mathrm{L}$, $3.91 \mathrm{mmol}$ ). The resulting solution was stirred at $0{ }^{\circ} \mathrm{C}$ for $30 \mathrm{~min}$, and then diluted with $\mathrm{CH}_{2} \mathrm{Cl}_{2} /$ aq $\mathrm{NaHCO}_{3}(20 \mathrm{~mL} / 30 \mathrm{~mL})$. The layers were separated and the aqueous layer was further extracted with $\mathrm{CH}_{2} \mathrm{Cl}_{2}(2$ $\times 15 \mathrm{~mL})$. The organic layers were combined, dried $\left(\mathrm{MgSO}_{4}\right)$, filtered, and concentrated to afford a residue that was used without purification in the next step. To the crude mesylate (obtained from the previous step) dissolved in toluene $(20 \mathrm{~mL})$ was added DBU $(5 \mathrm{~mL})$. The mixture was heated to reflux for $5 \mathrm{~h}$. The reaction mixture was allowed to cool to r.t. after which it was diluted with EtOAc/sat. aq $\mathrm{NH}_{4} \mathrm{Cl}$ $(20 \mathrm{~mL} / 20 \mathrm{~mL})$. The layers were separated and the aqueous layer was further extracted with EtOAc $(2 \times 20 \mathrm{~mL})$. The organic layers were combined, dried $\left(\mathrm{MgSO}_{4}\right)$, filtered, and concentrated to afford a residue that was chromatographed on silica gel using hexanes/EtOAc as eluent (2:1) to afford the product 4 as an oil; yield: $850 \mathrm{mg}$ (63\% over 2 steps); $[\alpha]_{\mathrm{D}}^{20}-60.2\left(c=17.9, \mathrm{CH}_{2} \mathrm{Cl}_{2}\right) ; R_{f}=0.44(2: 1$ hexanes/EtOAc). IR (film): 2952, 2857, 1739, 1713, 1625, 1506, 1233, 1075, $836 \mathrm{~cm}^{-1}$.

${ }^{1} \mathrm{H}$ NMR $\left(300 \mathrm{MHz}, \mathrm{CDCl}_{3}\right): \delta=6.78(\mathrm{~d}, J=8.4 \mathrm{~Hz}, 1 \mathrm{H}), 6.74(\mathrm{~d}, J=8.4$ $\mathrm{Hz}, 1 \mathrm{H}), 4.81(\mathrm{dd}, J=2.4,3.0 \mathrm{~Hz}, 1 \mathrm{H}), 4.43(\mathrm{t}, J=5.4 \mathrm{~Hz}, 1 \mathrm{H}), 4.18(\mathrm{t}$, $J=3.3 \mathrm{~Hz}, 1 \mathrm{H}), 4.01-4.05(\mathrm{~m}, 1 \mathrm{H}), 3.82-3.89(\mathrm{~m}, 1 \mathrm{H}), 3.85(\mathrm{~s}, 3 \mathrm{H})$, $3.27(\mathrm{~s}, 6 \mathrm{H}), 2.65(\mathrm{~d}, J=5.4 \mathrm{~Hz}, 2 \mathrm{H}), 2.39-2.58(\mathrm{~m}, 2 \mathrm{H}), 2.24-2.29(\mathrm{~m}$, $1 \mathrm{H}), 2.03-2.15$ (m, $2 \mathrm{H}), 1.93$ (s, $3 \mathrm{H}), 1.77-1.86(\mathrm{~m}, 1 \mathrm{H}), 0.85(\mathrm{~s}, 9 \mathrm{H})$, $0.08(\mathrm{~s}, 3 \mathrm{H}), 0.06(\mathrm{~s}, 3 \mathrm{H})$.

${ }^{13} \mathrm{C} \mathrm{NMR}\left(75 \mathrm{MHz}, \mathrm{CDCl}_{3}\right): \delta=207.8,170.7,148.9,142.9,127.1,126.0$, 123.0, 112.8, 104.7, 91.7, 67.1, 61.0, 59.2, 55.9, 53.4, 53.1, 34.3, 33.2, $32.7,25.5,24.4,20.8,17.9,-4.9,-5.1$.

MS (EI+): $m / z(\%)=522(20), 490$ (100), 430 (40), 373 (50), 341 (95), 313 (50), 199 (75), 111 (38).

HRMS (EI+): $m / z$ calcd for $\mathrm{C}_{27} \mathrm{H}_{42} \mathrm{O}_{8} \mathrm{Si}$ : 522.2649; found: 522.2622 .

\section{2-\{(3S,3aS,3a1R,9S,9aR)-3-[(tert-Butyldimethylsilyl)oxy]-9,9a- dihydroxy-5-methoxy-1,2,3,3a,3a1,8,9,9a-octahydrophenanthro- [4,5-bcd]furan-3a1-yl\}ethyl Acetate (3)}

To a stirred solution of keto acetal $4(440 \mathrm{mg}, 0.84 \mathrm{mmol})$ in toluene $(10 \mathrm{~mL})$ was added $50 \%$ aq TFA $(1.0 \mathrm{~mL})$. The biphasic mixture was heated to $50{ }^{\circ} \mathrm{C}$ for $30 \mathrm{~min}$, then it was allowed to cool down to r.t. The 
reaction mixture was diluted with EtOAc/sat. aq $\mathrm{NaHCO}_{3}(10 \mathrm{~mL} / 5$ $\mathrm{mL}$ ). The layers were separated and the aqueous phase was further extracted with EtOAc $(2 \times 10 \mathrm{~mL})$. The organic layers were combined, dried $\left(\mathrm{MgSO}_{4}\right)$, filtered, and concentrated to afford a residue that was used as crude in the next step. To the crude keto acetal (obtained from the previous step) dissolved in THF $(3 \mathrm{~mL})$ at $-78{ }^{\circ} \mathrm{C}$ was added $\mathrm{SmI}_{2}$ (14 mL, $0.1 \mathrm{M}$ in THF, $1.40 \mathrm{mmol}$ ). The resulting deep blue reaction mixture was stirred at $-78{ }^{\circ} \mathrm{C}$ for $30 \mathrm{~min}$. The cooling bath was removed and the mixture was diluted with EtOAc/sat. aq $\mathrm{NaHCO}_{3}(20$ $\mathrm{mL} / 15 \mathrm{~mL}$ ). The biphasic mixture was allowed to warm up to r.t., then the layers were separated. The aqueous layer was further extracted with EtOAc $(3 \times 20 \mathrm{~mL})$. The organic layers were combined, dried $\left(\mathrm{MgSO}_{4}\right)$, filtered, and concentrated to afford a residue that was chromatographed on silica gel using hexanes/EtOAc as eluent (2:1) to afford the product 3 as an oil; yield: $262 \mathrm{mg}$ (65\% yield over 2 steps); $[\alpha]_{\mathrm{D}}^{20}+41.5\left(c=0.9, \mathrm{CH}_{2} \mathrm{Cl}_{2}\right) ; R_{f}=0.33(1: 1$ hexanes/EtOAc).

IR (film): 3435, 2952, 2930, 2855, 1736, 1713, 1632, 1504, 1257, 1030 $\mathrm{cm}^{-1}$.

${ }^{1} \mathrm{H}$ NMR $\left(300 \mathrm{MHz}, \mathrm{CDCl}_{3}\right): \delta=6.72(\mathrm{~d}, J=8.1 \mathrm{~Hz}, 1 \mathrm{H}), 6.61(\mathrm{~d}, J=8.1$ $\mathrm{Hz}, 1 \mathrm{H}), 4.80$ (dd, $J=3.3,1.2 \mathrm{~Hz}, 1 \mathrm{H}), 4.18-4.27$ (m, $1 \mathrm{H}), 3.95-4.05$ (m, $1 \mathrm{H}), 3.97-4.03(\mathrm{~m}, 1 \mathrm{H}), 3.88-3.91(\mathrm{~m}, 1 \mathrm{H}), 3.86(\mathrm{~s}, 3 \mathrm{H}), 3.28(\mathrm{dd}$, $J=8.1,17.0 \mathrm{~Hz}, 1 \mathrm{H}), 3.02(\mathrm{dd}, J=3.0,17.0 \mathrm{~Hz}, 1 \mathrm{H}), 2.47-2.55(\mathrm{~m}, 1 \mathrm{H})$, 2.21-2.31 (m, $1 \mathrm{H}), 1.96(\mathrm{~s}, 3 \mathrm{H}), 1.65-1.71(\mathrm{~m}, 1 \mathrm{H}), 1.56-1.60(\mathrm{~m}, 1$ H), 1.30-1.36 (m, $1 \mathrm{H}), 1.05-1.13(\mathrm{~m}, 1 \mathrm{H}), 0.96(\mathrm{~s}, 9 \mathrm{H}), 0.21(\mathrm{~s}, 3 \mathrm{H})$, $0.16(\mathrm{~s}, 3 \mathrm{H})$.

${ }^{13} \mathrm{C}$ NMR $\left(75 \mathrm{MHz}, \mathrm{CDCl}_{3}\right): \delta=170.0,145.8,142.4,130.2,124.8,119.8$, 113.7, 91.6, 74.5, 73.6, 69.4, 61.8, 56.4, 49.8, 33.9, 33.8, 31.7, 25.7, $22.7,20.9,18.0,-5.0,-5.2$.

MS (EI+): $m / z(\%)=361$ (90), 343 (100), 315 (60), 305 (95), 287 (40), $249(80)$.

HRMS (EI+): $m / z$ calcd for $\mathrm{C}_{25} \mathrm{H}_{38} \mathrm{O}_{7} \mathrm{Si}$ : 478.2387; found: 478.2391 .

\section{2-\{(5aS,8aR,8a1R,11S,11aS)-11-[(tert-Butyldimethylsilyl)oxy]-2- methoxy-7-oxo-5a,8a1,9,10,11,11a-hexahydro-5H-furo[2',3',4',5':4,5]- phenanthro[8a,9-d][1,3]dioxol-8a1-yl)ethyl Acetate (12)}

To a stirred solution of diol 3 (100 mg, $0.21 \mathrm{mmol})$ in toluene $(3 \mathrm{~mL})$ was added carbonyldiimidazole $(102 \mathrm{mg}, 0.63 \mathrm{mmol})$. The resulting mixture was heated to $80^{\circ} \mathrm{C}$ for $3 \mathrm{~h}$, then allowed to cool down to r.t. The solvent was removed by rotary evaporation and the residue was chromatographed on silica gel using hexanes/EtOAc (2:1) as eluent to afford the carbonate 12 as an oil; yield: $75 \mathrm{mg}(71 \%) ;[\alpha]_{D}{ }^{20}+51.6(c=$ $\left.1.0, \mathrm{CH}_{2} \mathrm{Cl}_{2}\right) ; R_{\mathrm{f}}=0.65$ ( $1: 1$ hexanes/EtOAc).

IR (film): 3436, 2953, 2931, 2856, 1806, 1738, 1637, 1235, $1067 \mathrm{~cm}^{-1}$. ${ }^{1} \mathrm{H} \mathrm{NMR}\left(300 \mathrm{MHz}, \mathrm{CDCl}_{3}\right): \delta=6.79(\mathrm{~d}, J=8.1 \mathrm{~Hz}, 1 \mathrm{H}), 6.69(\mathrm{~d}, J=8.1$ $\mathrm{Hz}, 1 \mathrm{H}), 4.67(\mathrm{~d}, J=6.3 \mathrm{~Hz}, 1 \mathrm{H}), 4.06-4.12(\mathrm{~m}, 1 \mathrm{H}), 3.86(\mathrm{~s}, 3 \mathrm{H})$, 3.69-3.73 (m, $1 \mathrm{H}), 3.39-3.43(\mathrm{~m}, 1 \mathrm{H}), 3.42(\mathrm{dd}, J=8.1,15.3 \mathrm{~Hz}, 1 \mathrm{H})$, $2.95(\mathrm{dd}, J=8.1,15.3 \mathrm{~Hz}, 1 \mathrm{H}), 2.14-2.19(\mathrm{~m}, 1 \mathrm{H}), 2.00-2.11(\mathrm{~m}, 1 \mathrm{H})$, 1.88 (s, $3 \mathrm{H}), 1.69-1.84$ (m, $2 \mathrm{H}), 1.33-1.43$ (m, $1 \mathrm{H}), 0.90$ (s, $9 \mathrm{H}), 0.14$ $(\mathrm{s}, 3 \mathrm{H}), 0.05(\mathrm{~s}, 3 \mathrm{H})$.

${ }^{13} \mathrm{C}$ NMR $\left(75 \mathrm{MHz}, \mathrm{CDCl}_{3}\right): \delta=170.6,145.5,144.5,127.4,120.4,120.3$, $115.8,96.7,86.2,82.4,74.1,60.3,56.3,50.0,36.0,33.0,30.9,26.4$, 25.8, 25.6, 20.7, 18.0, -4.6, -5.0.

MS (EI+): $m / z(\%)=447$ (70), 419 (35), 387 (40), 343 (100), 313 (40), 269 (35), 117 (25).

HRMS (EI+): $m / z$ calcd for $\mathrm{C}_{26} \mathrm{H}_{36} \mathrm{O}_{8} \mathrm{Si}$ : 504.2179; found: 504.2172 .
$N-(2-\{(5 a S, 8 a R, 8 a 1 R, 11 S, 11 a S)-11-[($ tert-Butyldimethylsilyl)oxy]2-methoxy-7-oxo-5a,8a1,9,10,11,11a-hexahydro-5H-furo[2',3',4',5':4,5]phenanthro[8a,9-d][1,3]dioxol-8a1-yl\}ethyl)- $N, 4-d i m e t h y l b e n-$ zenesulfonamide (13)

To as stirred solution of carbonate 12 (50 mg, $0.10 \mathrm{mmol}$ ) in $\mathrm{MeOH}$ (2 $\mathrm{mL}$ ) was added $\mathrm{K}_{2} \mathrm{CO}_{3}(50 \mathrm{mg}, 0.36 \mathrm{mmol})$. The resulting suspension was stirred at r.t. for $2 \mathrm{~h}$. The solvent was removed by rotary evaporation and the residue was diluted with EtOAc/sat. aq $\mathrm{NaHCO}_{3}(10 \mathrm{~mL} / 5$ $\mathrm{mL}$ ). The layers were separated and the aqueous phase was further extracted with EtOAc $(2 \times 10 \mathrm{~mL})$. The organic layers were combined, dried $\left(\mathrm{MgSO}_{4}\right)$, filtered, and concentrated to afford a residue that was used as crude in the next step. To the crude alcohol (obtained from the previous step) and $\mathrm{N}$-methyl $p$-toluenesulfonyl amide (20 mg, $0.11 \mathrm{mmol})$ in THF $(1.5 \mathrm{~mL})$ at $-10{ }^{\circ} \mathrm{C}$ was added $n \mathrm{Bu}_{3} \mathrm{P}(35 \mu \mathrm{L}, 0.14$ $\mathrm{mmol})$, followed by TMAD $(23 \mathrm{mg}, 0.13 \mathrm{mmol})$. The reaction mixture was allowed to warm up to r.t. and stirred for $16 \mathrm{~h}$ at r.t. The solvent was removed by rotary evaporation to afford a residue that was used without purification in the next step. To the crude tosylamide carbonate (obtained from the previous step) dissolved in $\mathrm{MeOH}(2 \mathrm{~mL})$ was added aq $3 \mathrm{~N} \mathrm{NaOH}(0.5 \mathrm{~mL})$. The resulting cloudy solution was stirred at r.t. for $30 \mathrm{~min}$. The solvent was removed by rotary evaporation and the residue was diluted with EtOAc/ $\mathrm{H}_{2} \mathrm{O}(10 \mathrm{~mL} / 5 \mathrm{~mL})$. The aqueous layer was further extracted with EtOAc $(2 \times 10 \mathrm{~mL})$. The organic layers were combined, dried $\left(\mathrm{MgSO}_{4}\right)$, filtered, and concentrated to afford a residue that was chromatographed on silica gel using hexanes/EtOAc (2:1) as eluent to afford the product $\mathbf{1 3}$ as an oil; yield: $40 \mathrm{mg}(67 \%$ yield over 3 steps $) ;[\alpha]_{\mathrm{D}}^{20}+18.4\left(c=1.1, \mathrm{CH}_{2} \mathrm{Cl}_{2}\right) ; R_{f}=0.50(1: 1$ hexanes/EtOAc).

IR (film): 3466, 2928, 2855, 1633, 1505, $1159 \mathrm{~cm}^{-1}$.

${ }^{1} \mathrm{H} \mathrm{NMR}\left(300 \mathrm{MHz}, \mathrm{CDCl}_{3}\right): \delta=7.61(\mathrm{~d}, J=8.4 \mathrm{~Hz}, 1 \mathrm{H}), 7.27(\mathrm{~d}, J=8.4$ $\mathrm{Hz}, 1 \mathrm{H}), 6.73(\mathrm{~d}, J=8.1 \mathrm{~Hz}, 1 \mathrm{H}), 6.61(\mathrm{~d}, J=8.1 \mathrm{~Hz}, 1 \mathrm{H}), 4.75(\mathrm{~s}, 1 \mathrm{H})$, $4.71(\mathrm{~d}, J=3.0 \mathrm{~Hz}, 1 \mathrm{H}), 3.89-3.93(\mathrm{~m}, 2 \mathrm{H}), 3.87(\mathrm{~s}, 3 \mathrm{H}), 3.56(\mathrm{~d}, J=4.8$ $\mathrm{Hz}, 1 \mathrm{H}), 3.50(\mathrm{t}, J=6.6 \mathrm{~Hz}, 1 \mathrm{H}), 3.38-3.40(\mathrm{~m}, 1 \mathrm{H}), 3.26(\mathrm{dd}, J=8.1$, $17.4 \mathrm{~Hz}, 1 \mathrm{H}), 2.99$ (dd, $J=3.0,17.4 \mathrm{~Hz}, 1 \mathrm{H}), 2.78(\mathrm{td}, J=4.8,12.0 \mathrm{~Hz}, 1$ $\mathrm{H}), 2.69(\mathrm{~s}, 3 \mathrm{H}), 2.42(\mathrm{~s}, 3 \mathrm{H}), 2.23-2.40(\mathrm{~m}, 2 \mathrm{H}), 1.46-1.61(\mathrm{~m}, 2 \mathrm{H})$, 1.39-1.46 (m, $2 \mathrm{H}), 0.96$ (s, $9 \mathrm{H}), 0.22(\mathrm{~s}, 3 \mathrm{H}), 0.15$ (s, $3 \mathrm{H})$.

${ }^{13} \mathrm{C} \mathrm{NMR}\left(75 \mathrm{MHz}, \mathrm{CDCl}_{3}\right): \delta=145.5,143.1,142.5,135.2,129.6,127.4$, $124.6,119.9,113.8,91.7,74.4,73.7,69.6,56.5,49.7,46.9,34.9,33.9$, $33.8,31.8,25.7,22.9,21.5,18.0,-5.0,-5.2$.

MS (EI+): $m / z(\%)=603$ (20), 546 (30), 528 (10), 343 (12), 313 (12), 198 (100).

HRMS (EI+): $m / z$ calcd for $\mathrm{C}_{31} \mathrm{H}_{45} \mathrm{NO}_{7} \mathrm{SSi}$ : 603.2686; found: 603.2679 .

$N-\left(2-\left\{\left(3 S, 3 a S, 3 a^{1} R, 9 a S\right)-3-[(\right.\right.$ tert-Butyldimethylsilyl)oxy]-9a-hydroxy-5-methoxy-1,2,3,3a,3a',9a-hexahydrophenanthro[4,5bcd]furan-3a ${ }^{1}$-yl\}ethyl)- $\mathbf{N , 4 - d i m e t h y l b e n z e n e s u l f o n a m i d e ~ ( 2 ) ~}$

To a stirred solution of diol 13 (150 mg, $0.25 \mathrm{mmol})$ in $\mathrm{CH}_{2} \mathrm{Cl}_{2}(5 \mathrm{~mL})$ at $0{ }^{\circ} \mathrm{C}$ was added $\mathrm{NEt}_{3}(63 \mu \mathrm{L}, 0.50 \mathrm{mmol})$ followed by $\mathrm{MsCl}(29 \mu \mathrm{L}$, $0.37 \mathrm{mmol}$ ). The resulting solution was stirred at $0{ }^{\circ} \mathrm{C}$ for $30 \mathrm{~min}$, and then it was diluted with $\mathrm{CH}_{2} \mathrm{Cl}_{2} /$ aq $\mathrm{NaHCO}_{3}(10 \mathrm{~mL} / 10 \mathrm{~mL})$. The layers were separated and the aqueous layer was further extracted with $\mathrm{CH}_{2} \mathrm{Cl}_{2}(2 \times 10 \mathrm{~mL})$. The organic layers were combined, dried $\left(\mathrm{MgSO}_{4}\right)$, filtered, and concentrated to afford a residue that was used without purification in the next step. To the crude mesylate (obtained from the previous step) dissolved in toluene $(3 \mathrm{~mL})$ was added DBU $(1 \mathrm{~mL})$. The mixture was heated to reflux for $1 \mathrm{~h}$. The mixture was allowed to cool to r.t. after which it was diluted with EtOAc/sat. aq $\mathrm{NH}_{4} \mathrm{Cl}(10 \mathrm{~mL} /$ $10 \mathrm{~mL}$ ). The layers were separated and the aqueous phase was further extracted with EtOAc $(2 \times 10 \mathrm{~mL})$. The organic layers were combined, dried $\left(\mathrm{MgSO}_{4}\right)$, filtered, and concentrated to afford a residue that was 
chromatographed on silica gel using hexanes/EtOAc (2:1) as eluent to afford the product 2 as a viscous oil; yield: $101 \mathrm{mg}$ (70\% over 2 steps); $[\alpha]_{D}{ }^{20}+59.98\left(c=0.3, \mathrm{CHCl}_{3}\right) ; R_{f}=0.6(2: 1$, hexanes/EtOAc).

IR (neat): 3511, 2952, 2927, 2854, 1742, 1631, 1598, 1505, 1333, $1272,1157,1115 \mathrm{~cm}^{-1}$.

${ }^{1} \mathrm{H}$ NMR $\left(300 \mathrm{MHz}, \mathrm{CDCl}_{3}\right): \delta=7.53(\mathrm{~d}, J=8.2 \mathrm{~Hz}, 2 \mathrm{H}), 7.25(\mathrm{~d}, J=8.2$ $\mathrm{Hz}, 2 \mathrm{H}), 6.69(\mathrm{~d}, J=8.0 \mathrm{~Hz}, 1 \mathrm{H}), 6.61(\mathrm{~d}, J=8.0 \mathrm{~Hz}, 1 \mathrm{H}), 6.25(\mathrm{~d}, J=9.6$ $\mathrm{Hz}, 1 \mathrm{H}), 5.62(\mathrm{~d}, J=9.6 \mathrm{~Hz}, 1 \mathrm{H}), 4.47(\mathrm{~d}, J=6.3 \mathrm{~Hz}, 1 \mathrm{H}), 3.87(\mathrm{~s}, 3 \mathrm{H})$, 3.53 (ddd, $J=10.9,6.1,4.5 \mathrm{~Hz}, 1 \mathrm{H}), 3.04-2.96(\mathrm{~m}, 2 \mathrm{H}), 2.62(\mathrm{~s}, 3 \mathrm{H})$, 2.40 (s, $3 \mathrm{H}), 2.18-2.03(\mathrm{~m}, 1 \mathrm{H}), 2.02-1.89(\mathrm{~m}, 1 \mathrm{H}), 1.80-1.59(\mathrm{~m}, 2$ H), 1.52-1.40 (m, $2 \mathrm{H}), 0.90$ (s, $9 \mathrm{H}), 0.13(\mathrm{~s}, 3 \mathrm{H}), 0.04(\mathrm{~s}, 3 \mathrm{H})$.

${ }^{13} \mathrm{C}$ NMR $\left(75 \mathrm{MHz}, \mathrm{CDCl}_{3}\right): \delta=145.4,144.7,143.2,137.2,135.1,129.7$, 129.6, 127.5, 123.6, 123.2, 118.2, 113.8, 97.3, 75.8, 73.3, 56.7, 50.3, $46.9,35.2,34.4,33.0,25.9,25.2,21.6,18.2,-4.5,-4.9$.

MS (EI+): $m / z(\%)=436$ (62), 432 (59), 416 (43), 374 (39), 225 (26), 198 (23), 157 (24), 125 (40), 93 (28), 71 (26), 57 (100).

HRMS (EI+): $m / z$ calcd for $\mathrm{C}_{31} \mathrm{H}_{44} \mathrm{NO}_{6} \mathrm{SSi}$ : 585.2580; found: 586.2647 .

(4S,4aR,7S,7aS,12bR)-7-[(tert-Butyldimethylsilyl)oxy]-9-methoxy3-methyl-2,3,4,4a,5,6,7,7a-octahydro-1H-4,12-methanobenzofuro[3,2-e]isoquinolin-4a-ol (14)

To a stirred solution of tosylamide $\mathbf{2}(50 \mathrm{mg}, 0.085 \mathrm{mmol})$ in THF ( 8 $\mathrm{mL})$ was added $t$-BuOH $(100 \mu \mathrm{L}, 1.05 \mathrm{mmol})$. The solution was cooled to $-78{ }^{\circ} \mathrm{C}$ and ammonia was condensed $(30 \mathrm{~mL})$ to the reaction mixture. $\mathrm{Li}(60 \mathrm{mg}, 8.65 \mathrm{mmol}$ ) was added in three portions over $10 \mathrm{~min}$. The resulting deep blue solution was stirred at $-78^{\circ}$ for $10 \mathrm{~min}$. It was quenched by the sequential addition of solid $\mathrm{NH}_{4} \mathrm{Cl}(4 \mathrm{~g}), \mathrm{MeOH}(20$ $\mathrm{mL}$ ), and sat. aq $\mathrm{NH}_{4} \mathrm{Cl}(20 \mathrm{~mL})$. The reaction mixture was allowed to warm up to r.t. after which it was diluted with $\mathrm{CH}_{2} \mathrm{Cl}_{2}$ /sat. aq $\mathrm{NH}_{4} \mathrm{Cl}$ $(30 \mathrm{~mL} / 30 \mathrm{~mL})$. The layers were separated and the aqueous phase was further extracted with $\mathrm{CH}_{2} \mathrm{Cl}_{2}(2 \times 30 \mathrm{~mL})$. The organic layers were combined, dried $\left(\mathrm{MgSO}_{4}\right)$, filtered, and concentrated to afford a residue that was chromatographed on silica gel using $\mathrm{CH}_{2} \mathrm{Cl}_{2} / \mathrm{MeOH}(9: 1)$ as eluent to afford the product 14 as a solid; yield: $28 \mathrm{mg}(76 \%) ; \mathrm{mp}$ $120-122^{\circ} \mathrm{C}(\mathrm{MeOH}) ;[\alpha]_{\mathrm{D}}{ }^{20}+66.4\left(c=0.3, \mathrm{CH}_{2} \mathrm{Cl}_{2}\right) ; R_{f}=0.43(9: 1$ $\left.\mathrm{CH}_{2} \mathrm{Cl}_{2} / \mathrm{MeOH}\right)$.

IR (film): 3411, 2927, 2853, 1634, 1500, 1447, 1257, $1108 \mathrm{~cm}^{-1}$.

${ }^{1} \mathrm{H} \mathrm{NMR}\left(300 \mathrm{MHz}, \mathrm{CDCl}_{3}\right): \delta=6.75(\mathrm{~d}, J=8.1 \mathrm{~Hz}, 1 \mathrm{H}), 6.62(\mathrm{~d}, J=8.1$ $\mathrm{Hz}, 1 \mathrm{H}), 4.42(\mathrm{~d}, J=6.3 \mathrm{~Hz}, 1 \mathrm{H}), 3.89(\mathrm{~s}, 3 \mathrm{H}), 3.42-3.48(\mathrm{~m}, 1 \mathrm{H}), 3.13$ $(\mathrm{d}, J=18.3 \mathrm{~Hz}, 1 \mathrm{H}), 2.81(\mathrm{~d}, J=5.4 \mathrm{~Hz}, 1 \mathrm{H}), 2.59$ (dd, $J=5.4,18.3 \mathrm{~Hz}, 1$ H), $2.38(\mathrm{~s}, 3 \mathrm{H}), 2.17-2.22(\mathrm{~m}, 1 \mathrm{H}), 1.92-2.01(\mathrm{~m}, 1 \mathrm{H}), 1.55-1.58(\mathrm{~m}$, $2 \mathrm{H}), 1.42-1.46(\mathrm{~m}, 1 \mathrm{H}), 0.91(\mathrm{~s}, 9 \mathrm{H}), 0.13(\mathrm{~s}, 3 \mathrm{H}), 0.03(\mathrm{~s}, 3 \mathrm{H})$.

${ }^{13} \mathrm{C}$ NMR $\left(75 \mathrm{MHz}, \mathrm{CDCl}_{3}\right): \delta=144.3,143.9,132.6,125.3,118.3,114.8$, 96.5, 73.9, 70.3, 64.8, 57.0, 46.7, 45.6, 42.7, 30.4, 29.7, 27.4, 25.8, 22.0, $-4.6,-5.0$.

MS (EI+): $m / z(\%)=431(20), 374(100), 313(15), 157$ (15), 75 (20), 69 (35), 57 (20).

HRMS (EI+): $m / z$ calcd for $\mathrm{C}_{24} \mathrm{H}_{37} \mathrm{NO}_{4} \mathrm{Si}: 431.2492$; found: 431.2482 .

\section{(+)-Oxycodone [ent-(1)]}

To a stirred solution of ether 14 (15 mg, $0.035 \mathrm{mmol})$ in THF ( $1 \mathrm{~mL})$ was added TBAF $(174 \mu \mathrm{L}, 0.174 \mathrm{mmol})$. The reaction mixture was stirred at r.t. for $3 \mathrm{~h}$ after which it was diluted with EtOAc/ $\mathrm{H}_{2} \mathrm{O}(10$ $\mathrm{mL} / 3 \mathrm{~mL}$ ). The layers were separated and the aqueous layer was further extracted with $\mathrm{CH}_{2} \mathrm{Cl}_{2}(2 \times 10 \mathrm{~mL})$. The organic layers were combined, dried $\left(\mathrm{MgSO}_{4}\right)$, filtered, and concentrated to afford a residue that was used as crude in the next step. To the crude alcohol (obtained from the previous step) dissolved in $\mathrm{CH}_{2} \mathrm{Cl}_{2}(3 \mathrm{~mL})$ was added Dess-Martin periodinane $(60.6 \mathrm{mg}, 0.143 \mathrm{mmol})$. The reaction mix- ture was stirred at r.t. for $2 \mathrm{~h}$. It was diluted with sat. aq $\mathrm{Na}_{2} \mathrm{~S}_{2} \mathrm{O}_{3}(1$ $\mathrm{mL})$, followed by sat. aq $\mathrm{NaHCO}_{3}(1 \mathrm{~mL})$. The layers were separated and the aqueous phase was further extracted with $\mathrm{CH}_{2} \mathrm{Cl}_{2}(2 \times 10 \mathrm{~mL})$. The organic layers were combined, dried $\left(\mathrm{MgSO}_{4}\right)$, filtered, and concentrated to afford a residue that was chromatographed on silica gel using $\mathrm{CH}_{2} \mathrm{Cl}_{2} / \mathrm{MeOH}$ (9:1) as eluent to afford the product ent-(1) as a solid; yield: $6.5 \mathrm{mg}$ (59\% over 2 steps); $\mathrm{mp} 206-208{ }^{\circ} \mathrm{C}$ (Lit. ${ }^{12} \mathrm{mp}$ 207.4-209.5 ${ }^{\circ} \mathrm{C} ;[\alpha]_{\mathrm{D}}^{20}+205\left(c=0.3, \mathrm{CHCl}_{3}\right)\left\{\right.$ Lit. $^{12}[\alpha]_{\mathrm{D}}^{20}-207(c=$ $\left.0.09, \mathrm{CHCl}_{3}\right)$.

${ }^{1} \mathrm{H}$ NMR $\left(300 \mathrm{MHz}, \mathrm{CDCl}_{3}\right): \delta=6.69(\mathrm{~d}, J=8.1 \mathrm{~Hz}, 1 \mathrm{H}), 6.62(\mathrm{~d}, J=8.1$ $\mathrm{Hz}, 1 \mathrm{H}$ ), 4.66 (s, $1 \mathrm{H}$ ), 3.89 (s, $3 \mathrm{H}$ ), 3.15 (d, J = $18.6 \mathrm{~Hz}, 1 \mathrm{H}$ ), 3.01 (ddd, $J=5.1,14.4 \mathrm{~Hz}, 1 \mathrm{H}), 2.87(\mathrm{~d}, J=5.8 \mathrm{~Hz}, 1 \mathrm{H}), 2.55(\mathrm{dd}, J=5.8,18.6 \mathrm{~Hz}$, $1 \mathrm{H}), 2.40$ (s, $3 \mathrm{H}), 2.36-2.51(\mathrm{~m}, 2 \mathrm{H}), 2.28(\mathrm{dt}, J=3.3,14.4 \mathrm{~Hz}, 1 \mathrm{H})$, $2.12-2.18(\mathrm{~m}, 1 \mathrm{H}), 1.83-1.90(\mathrm{~m}, 1 \mathrm{H}), 1.64(\mathrm{dd}, J=3.3,14.4 \mathrm{~Hz}, 1 \mathrm{H})$, $1.55-1.60(\mathrm{~m}, 1 \mathrm{H})$.

${ }^{13} \mathrm{C}$ NMR $\left(75 \mathrm{MHz}, \mathrm{CDCl}_{3}\right): \delta=208.7,145.1,143.0,129.5,125.1,119.6$, 115.0, 90.5, 70.5, 64.7, 56.9, 50.3, 45.4, 42.8, 36.2, 31.5, 30.6, 22.1.

\section{Funding Information}

The authors are grateful to the following agencies for financial support of this work: Natural Sciences and Engineering Research Council of Canada (NSERC) (Idea to Innovation and Discovery Grants), Canada Research Chair Program, Canada Foundation for Innovation (CFI), TDC Research, Inc., TDC Research Foundation, the Ontario Partnership for Innovation and Commercialization (OPIC), Noramco, Inc., and The Advanced Biomanufacturing Centre (Brock University).

\section{Supporting Information}

Supporting information for this article is available online at https://doi.org/10.1055/s-0037-1611335. Included are spectral data for compounds $2,3,4,6,11,12,13,14$, ent-(1), 16.

\section{References}

(1) Jakubska, A.; Przado, D.; Steininger, M.; Aniol-Kwiatkowska, A.; Kadej, M. Appl. Ecol. Env. Res. 2005, 3, 29.

(2) (a) Lin, H.-C.; Wang, Z.; Boyd, C.; Simoni-Wastila, L.; Buu, A. Addict. Behav. 2018, 76, 348. (b) Li, Y.; Hong, R. A.; Robbins, B.; Gibbons, K. M.; Holman, A. E.; Caird, M. S.; Farley, F. A.; Abbott, M. D.; Burke, M. C. Spine 2018, 43, E98.

(3) https://www.rexall.ca/articles/view/894/Percocet.

(4) https://www.drugs.com/dosage/oxycontin.html.

(5) Chapman, R.; Rider, L. S.; Hong, Q.; Kyle, D.; Kupper, R. US Patent $7674800 B 2,2004$.

(6) (a) Freund, M.; Speyer, E. DE Patent 286431, 1914. (b) Freund, M.; Speyer, E. DE Patent 296916, 1916. (c) Freund, M.; Speyer, E. Angew. Chem. 1914, 27, 380. (d) Lutz, R. E.; Small, L. J. Org. Chem. 1939, 4, 220. (e) Kok, G. B.; Scammells, P. J. RSC Adv. 2012, 2 , 11318.

(7) (a) Millgate, A. G.; Pogson, B. J.; Wilson, I. W.; Kutchan, T. M.; Zenk, M. H.; Gerlach, W. L.; Fist, A. J.; Larkin, P. J. Nature 2004, 431, 413. (b) Fist, A. J.; Byrne, C. J.; Gerlach, W. L. US Patent 606749, 2000. (c) Fist, A. J. US Patent Application 20090227796 A1, 2009. (d) Thodey, K.; Galanie, S.; Smolke, C. D. Nat. Chem. Biol. 2014, 10, 837.

(8) https://tasalk.com.au/.

(9) Gates, M.; Tschudi, G. J. Am. Chem. Soc. 1952, 74, 1109. 
(10) (a) Rinner, U.; Hudlicky, T. Top. Curr. Chem. 2012, 309, 33. (b) Zezula, J.; Hudlicky, T. Synlett 2005, 388. (c) Taber, D. F.; Neubert, T. D.; Schlecht, M. F. In Strategies and Tactics in Organic Synthesis; Harmata, M., Ed.; Elsevier: London, 2004, 353. (d) Novak, B. H.; Hudlicky, T.; Reed, J. W.; Mulzer, J.; Trauner, D. Curr. Org. Chem. 2000, 4, 343. (e) Hudlicky, T.; Butora, G.; Fearnley, S.; Gum, A.; Stabile, M. In Studies in Natural Products Chemistry; Atta-ur-Rahman, Ed.; Elsevier: Amsterdam, 1996, 43.

(11) Rice, K. J. Org. Chem. 1980, 45, 3135.

(12) Kimishima, A.; Umihara, J.; Mizoguchi, A.; Yokoshima, S.; Fukuyama, T. Org. Lett. 2014, 16, 6244.

(13) Zylstra, G. J.; Gibson, D. T. J. Biol. Chem. 1989, 264, 14940

(14) For use of these metabolites in synthesis, see: (a) Taher, E. S.; Banwell, M. G.; Buckler, J. N.; Yan, Q.; Lan, P. Chem. Rec. 2018, 18, 239. (b) Banwell, M. G.; Bolte, B.; Buckler, J. N.; Chang, E. L.; Lan, P.; Taher, E. S.; White, L. V.; Willis, A. C. J. Proc. Royal Soc. New South Wales 2016, Parts 1 \& 2, 34. (c) Lewis, S. E. In Arene Chemistry: Reaction Mechanisms and Methods for Aromatic Compounds; Mortier, J., Ed.; Wiley-VCH: Weinheim, 2015, 915. (d) Lewis, S. E. Chem. Commun. 2014, 50, 2821. (e) Griffen, J. A.; Kenwright, S. J.; Abou-Shehada, S.; Wharry, S.; Moody, T. S.; Lewis, S. E. Org. Chem. Front. 2014, 1, 79. (f) Rinner, U. In Comprehensive Chirality; Carreira, E. M.; Yamamoto, H., Ed.; Elsevier: Amsterdam, 2012, 240. (g) Bon, J.-Y.; Lee, B.; Banwell, M. G.; Cade, I. A. Chim. Oggi Chem. Today 2012, 30, 22. (h) Hudlicky, T.; Reed, J. W. Synlett 2009, 685. (i) Hudlicky, T.; Reed, J. W. Chem. Soc. Rev. 2009, 38, 3117. (j) Boyd, D. R.; Bugg, T. D. H. Org. Biomol. Chem. 2006, 4, 181. (k) Johnson, R. A. Org. React. 2004, 63, 117. (1) Banwell, M. G.; Edwards, A. J.; Harfoot, K. A.; Jolliffe, K. A.; McLeod, M. D.; McRae, K. J.; Stewart, S. G.; Vogtle, M. Pure Appl. Chem. 2003, 75, 223. (m) Boyd, D. R.; Sharma, N. D. J. Mol. Catal. B: Enzym. 2002, 19-20, 31. (n) Boyd, D. R.; Sharma, N. D.; Allen, C. C. R. Curr. Opin. Biotechnol. 2001, 12, 564. (o) Hudlicky, T.; Gonzalez, D.; Gibson, D. T. Aldrichimica Acta 1999, 32, 35. (p) Boyd, D. R.; Sheldrake, G. N. Nat. Prod. Rep. 1998, 15, 309. (q) Reed, J. W.; Hudlicky, T. In Advances in Asymmetric Synthesis; Hassner, A., Ed.; JAI Press: Greenwich, 1995, 271. (r) Brow, S. M.; Hudlicky, T. In Organic Synthesis: Theory and Applications; Hudlicky, T., Ed.; JAI Press: Greenwich, 1993, 113. (s) Carless, H. A. J. Tetrahedron: Asymmetry 1992, 3, 795. (t) Widdowson, D. A.; Ribbons, D. W.; Thomas, D. D. Janssen Chim. Acta 1990, 8, 3.

(15) (a) Hudlicky, T.; Endoma, M. A. A.; Butora, G. J. Chem. Soc., Perkin Trans. 1 1996, 2187. (b) Endoma, M. A.; Bui, V. P.; Hansen, J.; Hudlicky, T. Org. Process Res. Dev. 2002, 6, 525.

(16) (a) Endoma-Arias, M. A. A.; Hudlicky, J. R.; Simionescu, R.; Hudlicky, T. Adv. Synth. Cat. 2014, 356, 333. (b) Hudlicky, T.; Endoma-Arias, M. A. A. Chem. Eur. J. 2016, 22, 14540.

(17) Koizumi, H.; Yokoshima, S.; Fukuyama, T. Chem. Asian J. 2010, 5 , 2191.

(18) Oxidation of the diol $\mathbf{3}$ to afford a keto alcohol followed by $\mathrm{NaBH}_{4}$ reduction afforded an epimeric diol. Attempts to form the acetonide of this supposed trans-isomer failed. The diol $\mathbf{3}$ was smoothly converted to the acetonide. Unpublished observations.
(19) (a) Parker, K. A.; Fokas, D. J. Am. Chem. Soc. 1992, 114, 9688. (b) Parker, K. A.; Fokas, D. J. Org. Chem. 2006, 71, 449.

(20) The outcome of the hydroamination was dependent on the condition of the reaction. Initial experiments resulted in the formation of an undesirable side product $\mathbf{1 5}$ (isolated as 16) from the reduction of the cinnamyl alcohol moiety (Scheme 3 ). The formation of this product was eliminated by reversing the order of addition of reagents. Thus, Li was added last in the reaction and in several portions (see experimental section). The same phenomenon has been observed originally by Birch $^{21}$ and later by Hall $^{22}$ in their studies with dissolving metal reduction of cinnamyl alcohols.

$N$-(2-\{(3S,3aS,3a1R,9aR)-3-[(tert-Butyldimethylsilyl)oxy]-9ahydroxy-5-methoxy-1,3,3a,8,9,9a-hexahydrophenanthro[4,5-bcd]furan-3a1(2H)-yl\}ethyl)- $N$,4-dimethylbenzenesulfonamide (16)

Mp 67-68 ${ }^{\circ} \mathrm{C}(\mathrm{MeOH}) ;[\alpha]_{\mathrm{D}}{ }^{20}+2.0\left(c=0.2, \mathrm{CH}_{2} \mathrm{Cl}_{2}\right) ; R_{f}=0.5(2: 1$ hexanes/EtOAc).

IR (film): 3500, 2925, 2854, 1735, 1600, 1461, 1338, 1257, 1160, $830 \mathrm{~cm}^{-1}$.

${ }^{1} \mathrm{H}$ NMR $\left(300 \mathrm{MHz}, \mathrm{CDCl}_{3}\right): \delta=7.57(\mathrm{~d}, J=8.4 \mathrm{~Hz}, 2 \mathrm{H}), 7.27(\mathrm{~d}$, $J=8.4 \mathrm{~Hz}, 2 \mathrm{H}), 6.76(\mathrm{~d}, J=8.1 \mathrm{~Hz}, 1 \mathrm{H}), 6.63(\mathrm{~d}, J=8.1 \mathrm{~Hz}, 1 \mathrm{H})$, 4.55 (d, J = 4.8 Hz, $1 \mathrm{H}), 3.87(\mathrm{~s}, 3 \mathrm{H}), 3.60(\mathrm{~m}, 1 \mathrm{H}), 3.34-3.38(\mathrm{~m}$, $1 \mathrm{H}), 2.84-2.97(\mathrm{~m}, 2 \mathrm{H}), 2.71(\mathrm{~s}, 3 \mathrm{H}), 2.65-2.72(\mathrm{~m}, 1 \mathrm{H}), 2.43$ (s, $3 \mathrm{H}), 2.12-2.21(\mathrm{~m}, 2 \mathrm{H}), 1.86-1.96(\mathrm{~m}, 1 \mathrm{H}), 1.40-1.81(\mathrm{~m}, 4$ $\mathrm{H}), 0.91(\mathrm{~s}, 9 \mathrm{H}), 0.14(\mathrm{~s}, 3 \mathrm{H}), 0.07(\mathrm{~s}, 3 \mathrm{H})$.

${ }^{13} \mathrm{C}$ NMR ( $75 \mathrm{MHz}, \mathrm{CDCl}_{3}$ ): $\delta=143.1,142.7,135.0,131.4,129.6$, 127.3, 125.4, 120.3, 114.3, 93.9, 77.2, 72.8, 71.5, 56.7, 49.7, 47.2, $35.2,33.0,31.8,25.8,25.0,24.3,21.5,18.0,-4.8,-5.2$.

MS (EI+): $m / z(\%)=587$ (10), 530 (20), 512 (25), 439 (25), 403 (20), 345 (40), 343 (70), 327 (50), 315 (45), 198 (100), 183 (30), 97 (25).

HRMS (EI+): $\mathrm{m} / z$ calcd for $\mathrm{C}_{31} \mathrm{H}_{45} \mathrm{NO}_{6} \mathrm{SSi}$ : 587.2737; found: 587.2726 .

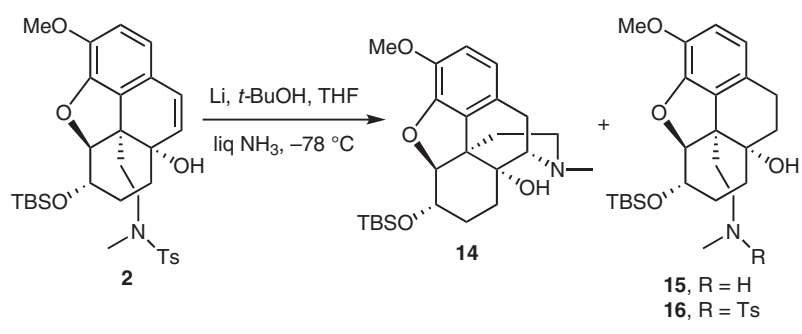

Scheme 3

(21) Birch, A. J. J. Chem. Soc. 1945, 809.

(22) Hall, S. S. J. Org. Chem. 1973, 38, 1738. 